\title{
Early detection of dermatological diseases in children admitted in a neurological unit: a retrospective study
}

\begin{abstract}
Background: Both the central nervous system and skin are derived from the same ectodermal origin during the embryogenesis, and thus children with neurologic disorders may have associated skin diseases, an area needs to spot light.

Objective: The aim of this study was to clarify and analyze the dermatological consultations encountered in a neurological unit.

Methods: Dermatological consultations during the last two years from children admitted in a neurological unit were retrospectively analyzed.

Results: Out of 1, 103 dermatology consultations, 318(28.8\%) came from the department of neurology. The ratio of male to female was 1: 0.7 . Consults were most frequently found from children with Encephalopathy $(25.8 \%)$. The most common dermatological consultations were Skin infections $(25.7 \%)$, Eczema/dermatitis $(31.5 .0 \%)$ followed by cutaneous adverse drug reactions $(10.8 \%)$.

Conclusion: This study clarifies the significant high frequency of skin diseases among hospital admitted children due to neurological illnesses. Integration between the pediatric neurologist and dermatologists as secondary care providers in one side and the family physician in the other side as primary care provider may improve the quality of patient care, help to early predict the occurrence of these illnesses and provide better outcome.
\end{abstract}

Keywords: dermatology, pediatric neurologist, referral, consultation

Volume I Issue2 - 2017

\author{
Mohammad Alkot,' Hani A Aziz Jokhdar, ${ }^{2}$ \\ Emad Eljahdaly, ${ }^{2}$ Mohammed A Garout, ${ }^{2}$ \\ Hossam Abdelbakiand, ${ }^{3}$ Jehad O Albitar ${ }^{4}$ \\ 'Department of Family Medicine, Menoufia University, Egypt \\ ${ }^{2}$ Department of Community Medicine \& Health Care of \\ Pilgrims, Umm Al-Qura university, Saudi Arabia \\ ${ }^{3}$ Department Pediatric, Menoufia University, Egypt \\ ${ }^{4}$ Umm Al-Qura University, Saudi Arabia
}

Correspondence: Mohammad Alkot, Department of Family medicine, Menoufia university, Egypt, Tel 540684479, Email Mohammed_elkott@yahoo.com

Received: November 17, 2016 | Published: May 15, 2017

\section{Introduction}

Skin disorders may be primary lesions or reflecting an underlying systemic illness. While dermatologic practice occurs primarily in the out-patient clinics, dermatologists also provide essential consultative services for in-patients admitted to other different disciplines. Several researches on the characteristics of dermatologic consultations have been reported. Several studies found that, patients from the neurology unit accounted for a significant portion of referrals as reported by Lyu et al. ${ }^{1}$ who also found the same result. ${ }^{1}$ However another studies showed that, the epidemiology of skin diseases among inpatient consultation has been described in the fields of obstetrics and gynecology, rheumatology, pediatricshematology, and the intensive care unit, in order of frequency. However, data describing dermatology consultations coming from neurology unit are limited. ${ }^{2,3}$

The epidermal and neuronal tissues are derived from a common neuroectodermal precursor. It is well known that, neurologic and dermatologic abnormalities occur concurrently in hereditary disorders such as tuberous sclerosis, neurofibromatosis, and xerodermapigmentosum. Moreover, much evidence has accumulated supporting an association between acquired neurological disturbances, Parkinsonism, or spinal cord injury and changes on skin such as seborrheic dermatitis (SD) or fungal infections occurring below the neurologic level of injury. ${ }^{4}$ The aim of this study was to clarify and analyze the dermatological consultations encountered in a neurological unit.

\section{Methods}

This was a retrospective study conducted at Banha specialized Pediatric hospital of Ministry of Health, Egypt. The hospital has a dermatology outpatient clinic. All cases either outpatients or referred inpatients were evaluated and reviewed by dermatology trainees and a senior consultant dermatologist. The diagnoses were made according to the International Classification of Disease, $10^{\text {th }}$ Revision.

All the in-patient referrals for dermatology consultations were reviewed between July 1, 2013 and June 30, 2015. Data were extracted from medical records and included the clinical record number, age, gender, requesting service, date of application, reason for admission to the hospital, and the final dermatological diagnosis. If the patient was not hospitalized, or had no apparent skin lesion or clinical symptoms related to the skin at the time of the visit, the case was excluded. Neurological conditions were divided into 9categories, as shown in (Table 2). Diagnoses made by the consulting dermatologist were divided into 12 categories (Table 3 ). In addition to the number of consultations, we also determined the relative frequency of consultations made for neurology unit in-patients compared with other specialties. 
Table I Demographic characteristics and clinical diagnosis recorded in reviewed files

\begin{tabular}{llll}
\hline Variable & Value & P-value & OR (95\% CI) \\
\hline Sex (n, \%) & & & \\
Male & $192(60.4)$ & 0.004 & $2.75(1.34 \sim 5.64)$ \\
Female & $126(39.6)$ & & \\
Age $(\mathbf{y r})$ & & & \\
Mean \pm SD & $9.8 \pm 6.7$ & 0.717 & \\
Median & 6.1 & & \\
Range & $2 \sim 17$ & & \\
Neurosurgical Conditions (n, \%) & & & \\
Encephalopathy & $82(25.8)$ & 0.048 & $1.96(1.00 \sim 3.85)$ \\
Cerebral infarction & $66(20.8)$ & 0.379 & $0.67(0.27 \sim 1.64)$ \\
Cerebral hemorrhage & $55(17.3)$ & 0.774 & $1.13(0.38 \sim 2.96)$ \\
Cerebral infection & $44(13.8)$ & 0.804 & $0.77(0.26 \sim 2.24)$ \\
Spinal fracture & $24(7.5)$ & 0.763 & $1.23(0.42 \sim 3.63)$ \\
Other spinal diseases & $18(5.7)$ & 1.000 & $0.67(0.15 \sim 2.91)$ \\
Brain tumor & $18(5.7)$ & 0.445 & $1.59(0.45 \sim 5.53)$ \\
Others & $11(3.4)$ & 0.710 & $0.46(0.06 \sim 3.46)$ \\
Total patients & $318(100.0)$ & & \\
\hline
\end{tabular}

Table 2 Frequency of In-patient services consulting dermatologist

\begin{tabular}{llll}
\hline Requesting service & Consultations & $\begin{array}{l}\text { Total Inpatients during the } \\
\text { study period }\end{array}$ & $\begin{array}{l}\text { Percentage of inpatients who are } \\
\text { referred to dermatology* }\end{array}$ \\
\hline Neurology & $318(28.8)$ & $932(8.9)$ & 34.1 \\
Pediatrics & $183(16.6)$ & $1231(11.7)$ & 14.9 \\
General surgery & $94(8.5)$ & $1123(10.8)$ & 8.4 \\
Orthopedics & $83(7.5)$ & $982(9.4)$ & 8.5 \\
Intensive care unit & $119(10.8)$ & $1532(14.6)$ & 7.8 \\
Otolaryngology & $35(3.2)$ & $388(3.7)$ & 9.1 \\
Cardiothoracic surgery & $63(5.7)$ & $605(5.8)$ & 10.4 \\
Urology & $36(3.3)$ & $879(8.4)$ & 4.1 \\
Family medicine & $130(11.8)$ & $1417(13.5)$ & 9.2 \\
Ophthalmology & $17(1.5)$ & $601(5.7)$ & 2.8 \\
Others & $25(2.3)$ & $789(7.5)$ & 3.2 \\
Total & $1103(100.0)$ & $10479(100.0)$ & 10.5 \\
\hline
\end{tabular}

Values are presented as number(\%). *(Total consults/total in-patients) $\times 100$.

Table 3 Recorded dermatological diagnosis among neurology unit' In-patient consulting dermatologist

\begin{tabular}{ll}
\hline Diagnosis & n (\%) \\
\hline Dermatitis & $108(31.5)$ \\
Contact dermatitis & $57(52.8)$ \\
Seborrhea dermatitis & $41(38.0)$ \\
Other Eczematous dermatitis & $10(9.2)$ \\
Infectious skin disorders & $88(25.7)$ \\
Fungal infection & $34(38.6)$ \\
Viral skin disease & $33(37.5)$ \\
Bacterial disease & $21(23.9)$ \\
Adverse Drug reaction - eruption & $37(10.8)$ \\
Urticaria & $24(7.0)$ \\
Folliculo-sebaceous diseases & $18(5.2)$ \\
Cutaneous vascular diseases & $13(3.8)$ \\
Hair disorders & $11(3.2)$ \\
Connective tissue diseases & $9(2.6)$ \\
Subcutaneous fat diseases & $8(2.3)$ \\
Pigment anomalies & $7(2.1)$ \\
Nail disorders & $5(1.4)$ \\
Miscellaneous* & $15(4.4)$ \\
Total & $343(100.0)$ \\
\hline
\end{tabular}




\section{Statistical analysis and ethics statement}

Statistical analyses were performed using Statistical Package for Social Sciences (SPSS) under windows using personal computer Comparisons of dermatologic variables between the groups (patients with specific neurosurgical disorders versus those with other diseases) were performed using chi-squared tests and Fisher's exact tests, as appropriate and $p$-values $<0.05$ were considered to be statistically significant. This study was approved by the ethical committee of the faculty of medicine, Menoufia University (Table 1).

\section{Discussion}

In recent years, the field of dermatology has evolved into a predominantly out-patient practice, with increasing emphasis on cosmetics and surgical procedures. ${ }^{4}$ While the number of patients admitted to dermatology services is decreasing, the need for dermatologists as consultants in the hospital setting is increasing. ${ }^{5}$

Although the type and number of referrals from other services varies according to factors such as the healthcare system and the proportion of beds allocated to each department, previous studies have reported a lower proportion of referrals from the department of neurology compared with our study results, accounting for $1.9 \%$, $3.0 \%$ and $11.9 \%$ of all referrals. Interestingly, one study found that, patients with neurological illnesses were the most frequently referred. Several other reports have shown that neurology patients also require frequent dermatology consults. ${ }^{6}$ This high number of consultations from neurology and/or neurosurgery patients likely reflects the fact that these patients are often bed-ridden and thus have a greater need for dermatologic care. ${ }^{7}$

Eczema and cutaneous infections were the two most common dermatologic disorders referred for management, accounting for over half of all consultations. This is consistent with previous findings. ${ }^{8}$ The two most common individual 'dermatitis' diagnoses encountered in this study were contact dermatitis and Seborrhea dermatitis. A previous report suggested that, sweating caused by inappropriate hospital room temperature, detergents used to clean bed clothes, and soaps are factors that can cause contact dermatitis. ${ }^{9}$ In addition, antiseptics in general, and specifically those used in surgical washing preparations, occlusive dressings for surgical wounds, confinement to the hospital bed for patients with delirium, athetotic movements or seizures, and use of catheters tend to contribute to dermatologic issues, particularly among neurology unit in-patients.

Reed et al. previously reported a high incidence of SD after spinal cord injury due to altered sebaceous secretion, dermatophytosis, and changes in sweat secretion. Similarly, Wilson et al. ${ }^{10}$ identified SD in $65 \%$ of recently injured quadriplegic patients. The accumulation of sebum and scales on inadequately scrubbed skin resulting from a prolonged period of immobilization were suggested as the possible cause of SD. In addition, the histopathologic findings of less differentiated sebaceous glands and thickened germinated layers in affected area of ipsilateral SD occurred after syringomyelia also suggest that a neurocutaneous mechanism may involve the sebaceous and immune pathways. ${ }^{10}$ But, among the neurosurgical disorders, Intracranial hemorrhage only has been significantly associated with Seborrheic dermatitis $(p=0.035)$. Proceeding from these findings and quadriplegic state seen in intracranial hemorrhage patients, it seems reasonable to assume that Seborrhea dermatitis is linked to abnormal neurologic condition of intracranial hemorrhage, just in overall perspective. However, there are no studies to address the specific relationship between intracranial hemorrhage and Seborrhea dermatitis.

Infectious skin disorders were one of the most common dermatologic diagnoses encountered in this study. Contributing factors for skin infection in neurosurgical in-patients are an altered skin barrier due to decreased sweat production, ${ }^{11}$ increased sebum excretion, ${ }^{12}$ and immunologic changes including a decrease in immune function via alterations in natural-killer cells, T-cells, and interleukin receptor levels. ${ }^{13}$ In contrast to a previous report that suggested an increased rate of fungal infections below the level of spinal cord injury, ${ }^{14}$ we did not identify a significant correlation between spinal disease and fungal skin infections. This discrepancy is possibly due to transfer of these patients to the rehabilitation medicine unit.

Adverse drug reactions was the third most frequent reason for consultation, which is consistent with that reported in previous studies. ${ }^{1,11,15}$ Notably, 37 patients $(10.8 \%)$ developed adverse drug reactions after exposure to contrast media, of which the most common adverse reaction was skin eruption, ${ }^{13}$ for computerized tomography or angiography. However, none of the individual neurosurgical conditions were significantly associated with adverse drug reactions except for cerebral aneurysm. There was a greater likelihood of an infectious neurosurgical condition in patients diagnosed with adverse drug reactions. The findings are natural as the major putative drugs implicated in cases of adverse drug reactions among dermatology referrals were antibiotics $(50 \%)$ and antiepileptics $(13 \%))^{13}$

The wide spectrum of dermatologic conditions seen in in-patient multidisciplinary hospitals can pose diagnostic and therapeutic challenges to non-dermatologists in patient care. Falanga et al., ${ }^{16}$ reported a mean diagnostic accuracy for dermatologic diagnoses made by non-dermatologists to be $48 \%$. Our results enhance understanding of in-patient neurosurgical dermatology consultations and may be helpful in developing educational materials and management guidelines. ${ }^{17}$

\section{Limitation of the study}

This study has some potential limitations. It has a retrospective design, with all the attendant limitations of a retrospective study. Reliance only on the medical records is potential limitation. Paucity of histological information limited the interpretation of data. Vagueness of a control group because the study was conducted in in-patients only. Comparing the dermatological findings in neurology unit' inpatients and the general population may make up for this weak point in case of need. And larger prospective studies may help to shed light on useful clinical features that may determine the relationship between underlying neurological disorders and dermatologic conditions such as ICH and SD.

\section{Acknowledgements}

None.

\section{Conflict of interest}

The author declares no conflict of interest. 


\section{References}

1. Lyu SM, Byun JY, Choi YW, et al. Clinical features of dermatologyconsulted inpatients: focus on the differences between individual departments. Korean J Dermatol. 2014;52(4):215-221.

2. McMahon P, Goddard D, Frieden IJ. Pediatric dermatology inpatient consultations: a retrospective study of 427 cases. J Am AcadDermatol. 2013;68(6):926-931.

3. Noh TK, Haw S, Won $\mathrm{CH}$, et al. A statistical analysis and clinical evaluation of dermatologic consultations in obstetrics and gynecologic inpatients Korean J Dermatol. 2012;50(11):951-958.

4. Tay LK, Lee HY, Thirumoorthy T, et al. Dermatology referrals in an East Asian tertiary hospital: a need for inpatient medical dermatology. Clin Exp Dermatol. 2011;36(2):129-134.

5. Chren MM. Dermatologic consultations--how can we know if we are effective? Initial methodologic considerations for the critical assessment of medical interventions performed by physicians. Arch Dermatol. 1994;130(8):1052-1054.

6. Fischer M, Bergert H, Marsch WC. The dermatologic consultation Hautarzt. 2004;55(6):543-548.

7. Koh H. A retrospective analysis of dermatological problems in a hematology ward. Clin Cosmet Investig Dermatol. 2013;6:145-149.

8. Lee HS, Yoo DJ, Park HW, et al. Is a stricter colonoscopy screening protocol necessary in liver transplant recipients? Comparison with an average-risk population. Dis Colon Rectum. 2014;57(8):976-982.
9. Penate Y, Guillermo N, Melwani P, et al. Dermatologists in hospital wards: an 8-year study of dermatology consultations. Dermatology. 2009;219(3):225-231.

10. Wilson CL, Walshe M. Incidence of seborrhoeic dermatitis in spinal injury patients. Br J Dermatol. 1988;119(33):48.

11. Yaggie JA, Niemi TJ, Buono MJ. Adaptive sweat gland response after spinal cord injury. Arch Phys Med Rehabil. 2002;83(6):802-805.

12. Thomas SE, Conway J, Ebling FJ, et al. Measurement of sebum excretion rate and skin temperature above and below the neurological lesion in paraplegic patients. Br J Dermatol. 1985;112(5):569-573.

13. Cruse JM, Lewis RE, Roe DL, et al. Facilitation of immune function, healing of pressure ulcers, and nutritional status in spinal cord injury patients. Exp Mol Pathol. 2000;68(1):38-54.

14. Chen TM, Fitzpatrick JE, Unilateral seborrheic dermatitis after decompression of Chiari I malformation and syringomyelia. J Am Acad Dermatol. 2006;55(2):356-357.

15. Choi MR, Park HS, Byun HJ, et al. Clinical evaluation of dermatology consultation in inpatients. Korean J Dermatol. 2010;48(3):163-170.

16. Falanga V, Schachner LA, Rae V, et al. Dermatologic consultations in the hospital setting. Arch Dermatol. 1994;130(8):1022-1025.

17. Jung KE, Chung J, Park BC, et al. A clinical study of cutaneous adverse reactions to nonionic contrast media in Korea. Ann Dermatol. 2012;24(1):22-25. 